\title{
PROVAS MAZE NA TRIAGEM DO RISCO NA LEITURA
}

\author{
Paula Marisa Fortunato Vaz ${ }^{1}$, Ana Paula Loução Martins ${ }^{2}$ and Luís de Miranda Correia ${ }^{3}$ \\ ${ }^{1}$ Instituto Politécnico de Bragança, Escola Superior de Educação; ${ }^{2}$ Centro de Investigação em Educação (CIEd), Instituto \\ de Educação, Universidade do Minho; ${ }^{3}$ Instituto de Educação, Universidade do Minho
}

Palavras-chave: Leitura, Provas Maze, Triagem Universal.

\begin{abstract}
No contexto de uma investigação mais ampla apresentam-se resultados de um estudo quantitativo que teve por objetivo testar a adequação técnica de uma prova de Monitorização Baseada no Currículo (Deno, 1985) para a compreensão da leitura. Nele participaram 82 alunos do $3^{\circ}$ ano de escolaridade, com 8 anos de idade, de um agrupamento de escolas Português. Concluiu-se que os resultados são fiáveis e válidos dados os coeficientes obtidos. Analisa-se ainda a utilização destas provas em Portugal, como instrumento de triagem, no contexto de um modelo preventivo baseado no grau de resposta à intervenção.
\end{abstract}

Perante a ausência, no sistema educativo Português, de mecanismos de monitorização do progresso dos alunos, assentes na utilização de provas validadas pela investigação no contexto de um modelo preventivo baseado no grau de resposta dos alunos à intervenção, tornou-se pertinente estudar a Monitorização com Base no Currículo (MBC) enquanto processo que permite, por um lado, identificar alunos em risco de Dificuldades de Aprendizagem Especificas (DAE) na leitura numa fase inicial da sua manifestação e, por outro, monitorizar o seu progresso ao longo do ano letivo. A MBC baseia-se no currículo nacional (Stecker, Sáenz, \& Lemons, 2007) e, através da utilização de provas de realização e administração breves, fáceis e estandardizadas, permite monitorizar o progresso dos alunos (Fuchs \& Fuchs, 2007b). Na área da leitura, mais especificamente para monitorizar a compreensão, são de uso comum nos EUA as provas Maze. Trata-se de provas normalmente realizadas em silêncio e em grupo (Brown-Chidsey, Davis, \& Maya, 2003) (também podem ser realizadas oralmente, o que exclui a possibilidade de realização em grupo) e cuja eficácia está comprovada por um grande corpo de investigação (Busch \& Lembke, 2005). O uso destas provas permite a identificação de alunos que no início do ano letivo possam estar em risco na leitura e que portanto necessitam de intervenção adicional, e também a sua monitorização durante todo o ano letivo bem como a tomada de decisões relativamente à intervenção e ao ensino (Shinn \& Shinn, 2002).
A MBC na leitura e a utilização de provas Maze têm sido sucessivamente validadas pela investigação (Busch \& Lembke, 2005) que tem vindo a ser realizada tendo por base o Modelo de Resposta à Intervenção (MRI) (Brown-Chidsey \& Steege, 2010; D. Fuchs, Fuchs, \& Vaughn, 2008; NCRI, 2012; Vaughn \& Bos, 2009). O MRI é um Modelo faseado de caráter preventivo que tem como principal objetivo prevenir e solucionar as dificuldades académicas e de comportamento proporcionando um ensino de sala de aula eficaz e intervenções de apoio de crescente intensidade, ao longo de diferentes níveis, validadas pela investigação que se iniciam na classe regular (Fletcher \& Vaughn, 2009; NRCLD, 2007). Assim, num primeiro momento, visa identificar e apoiar precocemente alunos que não progridem de forma adequada e que, portanto, podem estar em risco de apresentarem DAE e, num segundo momento, identificar alunos com DAE, como sendo aqueles que não respondem a intervenção/ensino validados e estandardizados e que, portanto, necessitam de uma intervenção individualizada dos serviços de educação especial (Fuchs \& Fuchs, 2007a).

Neste contexto desenvolvemos um estudo com o objectivo de testar a adequação técnica de uma prova de Monitorização Baseada no Currículo (Deno, 1985) para a compreensão da leitura.

\section{Metodologia}

\section{Participantes}

Neste estudo participaram 82 alunos (47 raparigas e 35 rapazes) sem NEE identificadas e sem apoio dos serviços de educação especial, que constituíam a população do $3^{\circ}$ ano de escolaridade de um agrupamento de escolas de Trás-os-Montes e Alto Douro-Portugal no ano letivo de 2010/2011. Estes alunos encontravam-se distribuídos por seis turmas.

\section{Instrumento de recolha de dados}

No contexto deste estudo construiu-se uma prova de MBC - Prova Maze, tendo por base as regras propostas por Busch e Lembke (2005) e por Shinn e Shinn (2002). A prova consiste num texto ao qual, com exceção da primeira frase, junto de cada sétima palavra foram 
acrescentadas duas outras palavras que não conferem sentido ao texto. Assim o aluno durante a leitura do texto, e ao longo de três minutos (Busch \& Lembke, 2005), tem de escolher de entre as três palavras aquela que faz sentido no texto (Brown-Chidsey, Davis, \& Maya, 2003). De referir que a prova foi realizada em cada turma, coletivamente. Para a cotação utilizou-se o procedimento de cotação "Número de respostas corretas menos metade das incorretas".

Antes da realização da prova pelos participantes no estudo fez-se um pré-teste numa turma do mesmo ano de escolaridade de um outro agrupamento de escolas, não se tendo verificado dificuldades por parte dos alunos que obrigassem a alterações na prova.

\section{Procedimentos de recolha e análise dos dados}

Depois de obtida a autorização para a realização do estudo no agrupamento de escolas, e mediante o consentimento dos pais e dos alunos, deu-se início ao processo de recolha de dados. Assim tiveram lugar, no ano letivo 2010/2011, uma primeira monitorização no outono, uma segunda monitorização já no inverno e uma terceira monitorização na primavera. No mesmo ano letivo, também na primavera, teve lugar uma avaliação com o Teste de Idade de Leitura (TIL).

Todas as monitorizações foram realizadas coletivamente, na presença do professor de cada turma e durante a aplicação seguiram-se as regras estandardizadas para a implementação da prova (adaptadas por Patrão, 2010, de Busch \& Lembke, 2005 e de Shinn \& Shinn, 2002).

Com vista ao estudo da fidelidade dos resultados obtidos, recorreu-se ao método Teste-reteste e calculou-se o coeficiente de correlação $r$ de Pearson (Almeida \& Freire, 2000) tendo por base os resultados obtidos quando utilizado o procedimento de cotação "Número de respostas corretas menos metade das incorretas".

A partir dos resultados obtidos na prova Maze na primavera, quando usado o mesmo procedimento de cotação, estudou-se também a sua validade correlacionando-se (recorrendo ao $r$ de Pearson) estes resultados com os resultados obtidos no TIL.

\section{Resultados}

Após o cálculo do $r$ de Pearson, entre a monitorização do outono $10 / 11$ e o reteste, obteve-se um valor indicativo de uma associação linear alta (.831) (Pestana \& Gageiro, 2008), significativo a $1 \%$, que remete portanto para a fiabilidade dos resultados obtidos com a prova Maze para este procedimento de cotação.

Relativamente à validade, o coeficiente de correlação obtido após o cálculo do $r$ de Pearson entre os resultados obtidos na prova Maze na primavera e os resultados no TIL, remete para uma associação linear alta (.794)
(Pestana \& Gageiro, 2008) e significativo a 1\%, o que remete também para a existência de validade dos resultados obtidos na prova Maze, com a utilização deste procedimento de cotação.

\section{Conclusões e Discussão}

Tal como em investigações internacionais (Espin, Wallace, Lembke, Campbell, \& Long, 2010; Pierce, McMaster, \& Deno, 2010; Tichá, Espin, \& Wayman, 2009) os resultados obtidos na prova Maze utilizada, são fiáveis e válidos para o procedimento de cotação usado "Número de respostas corretas menos metade das incorretas" dados os coeficientes de correlação encontrados, indicativos de associações lineares altas de acordo com Pestana e Gageiro (2008). Destaca-se ainda o facto de serem provas de realização breve e de fácil implementação e cotação.

Assim, parece legítimo pensar-se que as provas Maze podem ser usadas como instrumento de triagem, no sistema educativo Português, no contexto de um modelo preventivo baseado no grau de resposta dos alunos à intervenção. Desta forma a triagem universal realizada no outono, no inverno e na primavera, pode ser feita nos quatro anos de escolaridade, com recurso a provas Maze, para deteção de alunos em risco, aqueles que se encontram abaixo do percentil 20 (Deno et al., 2009). Esta deve ser seguida da implementação de programas de intervenção em diferentes níveis, para os alunos identificados como estando em risco e da monitorização semanal destes mesmos alunos no âmbito da implementação dos referidos programas.

\section{Conflicts of interest}

Não existem conflitos de interesses. Financiado pela FCT no âmbito do PROTEC: SFRH/PROTEC/67381/2010

\section{Address for correspondence}

Paula Marisa Fortunato Vaz

Escola Superior de Educação de Bragança

Campus de Santa Apolónia, Apartado 1101

5301 - 856 Bragança

Email:paulavaz@ipb.pt

\section{Referências bibliográficas}

Almeida, L. S. \& Freire, T. (2000) Metodologia da investigação em psicologia e educação. Braga: Psiquilíbrios.

Brown-Chidsey, R., Davis, L. \& Maya, C. (2003) 'Sources of variance in curriculum- based measures of silent reading.' Psychology in the Schools, 40 (4), pp. 363-77.

Brown-Chidsey, R. \& Steege, M. W. (2010) Response to intervention: Principles and strategies for effective practice.

Busch, T. W. \& Lembke, E. S. (2005) Teaching tutorial 5: Progress monitoring in reading using the $\mathrm{cbm}$ 
maze procedure. Division for Learning Disabilities of the Council for Exceptional Children.

Deno, S. L. (1985) 'Curriculum-based measurement: The emerging alternative.' Exceptional Children, 52 (3), pp. 219-32.

Deno, S.L., Reschly, A.L., Lembke, E.S., Magnusson, D., Callender, S.A., Windram, H. \& Stachel, N. (2009)

'Developing a school-wide progress-monitoring system.' Psychology in the Schools, 46 (1), pp. 45-55.

Espin, C., Wallace, T., Lembke, E., Campbell, H. \& Long, J. D. (2010) 'Creating a progress-monitoring system in reading for middle-school students: Tracking progress toward meeting high-stakes standards.' Learning Disabilities Research \& Practice, 25 (2), pp. 60-75.

Fletcher, J. M. \& Vaughn, S. (2009) 'Response to intervention: Preventing and remediating academic difficulties.' Child Development Perspectives, 3, pp. 30-7.

Fuchs, L. S. \& Fuchs, D. (2007a) 'A model for implementing responsiveness to intervention.' Teaching Exceptional Children, 39 (5), pp. 14-20.

Fuchs, L. S. \& Fuchs, D. (2007b) Using cbm for progress monitoring in reading. United States Office of Special Education Program; Student Progress Monitoring.

Fuchs, D., Fuchs, L. S. \& Vaughn, S. (eds) (2008) Response to intervention: A framework for reading educators. Newark: International reading association.

NCRI (2012) Multi-level prevention system. Retrieved january 1, 2012, from National Center on Response To Intervention Web site http://www.rti4success.org, from http://www.rti4success.org/categorycontents/ multi-level_prevention_system
NRCLD (2007) What is responsiveness to intervention? (brochura). Lawrence, KS: Autor.

Patrão, M. S. G. (2010) Monitorização com base no currciculo: Um estudo quantitativo sobre a utilização de provas maze no contexto do nível $i$ do modelo de atendimento à diversidade. (Tese de Mestrado não publicada), Universidade do Minho, Braga.

Pestana, M. H. \& Gageiro, J. N. (2008) Análise de dados para ciências sociais: A complementaridade do spss. Lisboa: Edições Sílabo.

Pierce, R., McMaster, K. \& Deno, S. L. (2010) 'The effects of using different procedures to score maze measures.' Learning Disabilities Research \& Practice (Blackwell Publishing Limited), 25 (3), pp. 151-60.

Shinn, M. R. \& Shinn, M. M. (2002) Aimsweb training workbook: Administration and scoring of reading maze for use in general outcome measurement. Retrieved 5-11- 2010 http://www.aimsweb.com/ uploads/pdfs/scoring_maze.pdf

Stecker, P. M., Sáenz, L. \& Lemons, C. (2007, 30-122010). [Introduction to using cbm for progress monitoring in reading].

Tichá, R., Espin, C. \& Wayman, M. M. (2009) 'Reading progress monitoring for secondary-school students: Reliability, validity, and sensitivity to growth of reading-aloud and maze-selection measures.' Learning Disabilities Research \& Practice, 24 (3), pp. 132-42.

Vaughn, S. \& Bos, C. (2009) Strategies for teaching students with learning and behavior problems (7th edn). New Jersey: Pearson. 